\title{
Cálculo de los costos de calidad y no calidad en empresas de salud y creación de un sistema de medición
}

\section{Calculation of Cost of Quality and No Quality in Health Firms and Creation of a Measuring System}

Luis Javier Arango C.* Carlos Iván Rodríguez M.** Rocío López C.***

\section{Resumen}

Con el objetivo central de convertir la gran cantidad de bibliografía existente en una herramienta práctica de utilidad diaria como apoyo a la gestión de las empresas de salud, se ha diseñado una metodología para el cálculo de los costos de calidad y no calidad en los procesos claves de la empresa de salud, aplicada en 2 hospitales piloto de Bogotá, en los cuales se crea un sistema de medición que se les deja como un valor agregado del proyecto, para que sistemáticamente tengan la cuantificación del monto de la inversión mensual en el mantenimiento y mejoramiento de la calidad de los procesos y los costos injustificados (no calidad) en los mismos procesos, ocasionados por fallas en las etapas críticas de cada proceso. Se busca entregar a cada hospital o empresa de salud, una herramienta que apoye la toma de decisiones y el sistema de gestión más acertado, sustentado en indicadores debidamente cuantificados mes a mes.

Los resultados de la medición de prueba realizada para el primer mes demostraron la significativa importancia de los costos de no calidad en los procesos monitorizados, injustificados por tratarse de fallas en los procesos claves de

\footnotetext{
* Médico. MSP. AAMH. Docente posgrados Universidad Santo Tomás, Bogotá D.C., Tunja y Bucaramanga. Creador de la metodología y el Sistema de medición de costos de calidad y no calidad en empresas de salud.

* Médico. Especialista en Auditoría y Calidad. Coinvestigador.

***** Economista. Investigadora asociada. Docente de posgrados, Universidad Santo Tomás, Bogotá D.C. y Tunja.
} 
la empresa, que afectan de forma importante la situación económica de la entidad, en un sector altamente competido y con unos márgenes de rentabilidad mínimos, por lo que su contención, se convierte en un apoyo para la sostenibilidad económica.

Palabras clave: costos de calidad, Costos de no calidad.

\section{Clasificación JEL: A12, I19}

\section{Abstract}

With the central objective to use the pertinent bibliography as a practical tool to support to the management of the companies of health, a methodology has been designed for the calculation of the Costs of Quality and Non Quality in the basic processes of the company, applied in 2 hospitals pilot of Bogotá D.C. For both of them was built a System of Mensuration as an added value of the project in order to provide a systematically form of quan-

\section{Introducción}

Basados en la extensa bibliografía al respecto y en la amplia experiencia de más de 20 años del grupo de investigadores en la gerencia de empresas y programas de salud $^{1}$, se diseñó una metodología para el cálculo de los costos de calidad y no calidad en los procesos claves de las empresas de salud, para lo cual se seleccionaron como pilotos, 2 hospitales del Distrito Capital, uno de 1er y 2do nivel de complejidad y otro de 3er nivel, en los que se encontraban como directivos egresados de la Universidad Santo Tomás de las especializaciones de Gerencia en

1 Diseño del estudio, metodología y sistema de medición creada por investigadores. tification for the monthly investment to maintain and improve the quality of the processes against the unjustified cost caused by flaws in the critical stages of each process. It is looked for surrender to each hospital or company of health a tool that supports the making decisions process and the System of Management for make right decisions, based on indicators properly quantified month to month.

The measurement results of test for the first month, demonstrated the negative impact of not monitored the Cost of Quality in processes, because of unjustified failure in the key processes of the company, significantly affecting the economic situation of the entity in a highly competitive sector with minimum profit margins, so the containment, it becomes a support for economic sustainability.

Key words: quality costs, costs of non quality.

Classification JEL: A12, I19

Instituciones de Seguridad Social y Auditoría de Salud. El estudio se programó para su realización en el segundo semestre de 2008.

Como un valor agregado para estos hospitales, el estudio en su segunda fase, creará y dejará en funcionamiento un Sistema de Medición, que permitirá a la Gerencia de cada hospital, cada mes, conocer el valor de la inversión en el mantenimiento y mejoramiento de la calidad (promoción y prevención) en los procesos claves para el funcionamiento de la entidad y el monto de los costos injustificados (de no calidad) por fallas o problemas en dichos procesos. 


\section{Los costos de calidad}

Toda empresa invierte sumas importantes en lograr que los servicios o productos que entrega a sus clientes tengan la calidad óptima, mínimo las características básicas que espera el cliente, esto con el fin de lograr su permanencia con la empresa y la seguridad al reducir los riesgos de problemas legales y reclamaciones por afectación del usuario.

En general, casi ninguna organización y especialmente en el Sistema General de Seguridad Social en Salud, realiza sistemáticamente la medición para conocer mes a mes, cuál es el monto invertido en mantener los estándares de calidad establecidos por la organización y exigidos por la legislación, situación que no permite que tengan en cuenta esta valiosa información, para apoyar la toma de decisiones de mejoramiento de la calidad y como base para definir el presupuesto asignado a los procesos dirigidos al logro de una calidad aceptada por los clientes y los entes de vigilancia y control.

Considerados estos costos por la mayoría de los autores como la inversión en promoción y prevención, las sumas invertidas en calidad deberían verse reflejadas en el mejoramiento de la satisfacción de los clientes, en la reducción de las quejas y los costos de operación injustificados o de no calidad.

\section{Los costos de no calidad}

Más relevante, por la importancia que puede tener para la sostenibilidad económica de la empresa, es conocer el monto de los costos (¿gastos?) ocasionados por la no calidad de los procesos establecidos, es decir, el valor en pesos gastado en solucionar las fallas en los procedimientos, por errores humanos, técnicos, ignorancia y negligencia, que obligan a la utilización de recursos no justificados, si las cosas se hubieran hecho bien desde la primera vez.
La medición sistemática de los costos de estas fallas, son:

- Repeticiones de servicios, productos, facturas, cuentas de cobro y programaciones de atenciones.

- Reprocesos por malos resultados de atenciones ambulatorias, quirúrgicas, de laboratorio clínico u otros servicios de apoyo.

- Rectificaciones y consumo de recursos humanos, físicos, tecnológicos, económicos para resolver quejas y reclamos directos o a través de juzgados.

- Inversión de recursos en resolución de problemas de mala calidad, como las infecciones nosocomiales, complicaciones quirúrgicas, anestésicas y de técnicas inadecuadas de esterilización, entre otras.

De esta manera se conoce el valor económico que sobre la empresa tienen tales problemas y sería la base de la toma de decisiones para el control de dichos costos, con lo que mes a mes se puede saber el impacto de las decisiones tomadas en forma cuantitativa.

Un adecuado control y mejoramiento de cada proceso, debería reducir los costos de no calidad, aquellos denominados fallas internas y externas.

Un buen sistema de medición de los costos de calidad y no calidad permite comparar mes a mes el efecto de la inversión en el mejoramiento y mantenimiento de la calidad (promoción y prevención), sobre los costos injustificados, de no calidad (fallas internas y externas), los cuales deberían reducirse significativamente a medida que invertimos en la obtención de la calidad.

La mala calidad le cuesta dinero a la economía de todo país, la diferencia entre un país y otro es el porcentaje en que afecta a sus ingresos. La mala calidad afecta sin distinguir si se trata de una empresa manufacturera, ministerio, banco, municipio, casino, empresa familiar, etc. En la empresa, su presencia se refleja en los estados financieros 
del período, finalmente esto afecta la caja fiscal debido a la disminución de las utilidades antes de impuestos. Lo paradójico es que no se llevan registros de las pérdidas producidas por la mala calidad, debido a que toda área trata a toda costa de ocultar sus ineficiencias. Si antes las empresas disimulaban su ineficiencia elevando precios y/o tarifas, esta práctica ya es desestimada porque ahora quien fija el precio y/o tarifa es el mercado (García \& Quispe \& Raez).

Si aceptamos la afirmación de los autores Carlos Colunga D. y Arturo Saldierna D., en su libro: Los costos de calidad (1994), la trascendencia para la sostenibilidad de las empresas y su rentabilidad es evidente: “...en las empresas estudiadas en Estados Unidos se encontró que entre del 25\% al 30\% de los precios de los productos y servicios se genera por costos debidos a no trabajar con calidad".

\section{El problema}

Las empresas de salud no conocen el monto de los dineros invertidos mensualmente en el mantenimiento y mejoramiento de la calidad de sus procesos, ni los costos injustificados por problemas en dichos procesos, por lo tanto no tienen un soporte adecuado para la eficaz toma de decisiones, dirigidas al mejoramiento de la calidad y el control de dichos costos.

\section{La hipótesis}

Las empresas de salud son organizaciones que tienen múltiples y disímiles procesos administrativos y asistenciales. Las fallas en los mismos, generan altos costos injustificados en la operación, que de ser cuantificados mes a mes, permitirán aplicar correctivos oportunos y, por ende, mejorar la competitividad de las mismas.

\section{Objetivos}

1. Identificar y medir los costos de los procesos establecidos para la implementación y mantenimiento del sistema de calidad en las empresas.

2. Identificar y medir los procedimientos que en cada uno de los procesos claves generan los principales problemas, considerados como costos de no calidad.

3. Crear un modelo para alimentar el subsistema de información y gestión de las empresas de salud, que permita estimar mensualmente el monto invertido en garantizar la calidad y, asimismo, cuantificar los costos de no calidad en los procesos críticos de los servicios y áreas prioritarias de la organización, como soporte para la toma de decisiones de mejoramiento y/o control.

\section{Justificación}

Un hecho evidente por toda la bibliografía nacional e internacional existente sobre los costos de calidad y no calidad, encontrados en las empresas y, algunos de los estudios de los propios investigadores al respecto, el monto es tan significativo que su medición permanente le da a la gerencia una información de gran valor como herramienta para la adopción de correctivos que le mejoren el control de los costos injustificados y la gestión.

Igualmente, cada mes tendrán en forma cuantitativa el resultado e impacto de las decisiones tomadas el mes anterior en contención de costos de no calidad, con respecto a la inversión en calidad.

\section{Alcance del proyecto}

1. El proyecto se desarrollará en su primera fase en dos instituciones prestadoras de servicios de salud de las más representativas del Distrito Capital, por su importancia como centro de referencia en la ciudad y 
ESE de 3er nivel de complejidad (Hospital Santa Clara) y en la zona central: ESE Hospital de Chapinero, ambas con directivos egresados de los programas de Gerencia de Instituciones de Seguridad Social de la Universidad Santo Tomás de Bogotá.

2. En su segunda fase de mercadeo e implementación podrá extenderse a todas las empresas de salud del país que reconozcan la importancia de conocer sus costos de calidad y los de no calidad, como bases para el control y mejoramiento de la gestión empresarial.

\section{Ingeniería del proyecto}

La construcción general del proyecto estableció la siguiente estructura y organización de la investigación, para la obtención de los productos proyectados:

1. Análisis de la fundamentación teórica.

2. Planteamiento del problema.

3. Formulación de la hipótesis a resolver con el estudio.

4. Comprobación de la hipótesis:

a. Selección de empresas para el estudio piloto.

b. Observación de los procesos y fenómenos que generan los costos de calidad y no calidad.

c. Cuantificación en costeo por proceso de dichos costos.

d. Análisis de resultados para evaluar la importancia de los resultados en la operación de la empresa.

e. Diseño de metodología para la medición sistemática de los costos de calidad y de no calidad en los procesos de las empresas de salud, como un modelo replicable en todas las empresas del sector de la seguridad social en el país.

f. Diseño de procesos para el mercadeo e implementación del modelo en el país.

g. Validación con directivos de las empresas de salud participantes y entrega de modelo a la Universidad.
Para el estudio se seleccionaron los siguientes procesos claves a cada uno de los cuales se les realizó evaluación técnica por el equipo investigador y se validaron sus resultados con los responsables de cada proceso y los directivos:

Contratación de servicios de salud.

1. Facturación.

2. Gestión de cartera.

3. Autorizaciones de servicios de salud.

4. Exámenes de laboratorio ineficaces.

5. Exámenes de radiología ineficaces.

6. Estancia hospitalaria (prolongada por infección Nosocomial).

7. Atención al usuario (quejas del cliente externo).

8. Atención de urgencias.

9. Formulación de medicamentos.

Para cada uno de ellos se diseñó y construyó una matriz para la recepción y procesamiento de la información y la producción de resultados.

El Sistema de medición de los costos de calidad y no calidad, tiene la siguiente estructura general:

1. Una matriz de datos para el registro de la información básica de las variables que permitirán en cada proceso:

- La cuantificación de lo invertido en la calidad del proceso (prevención e inspección).

- Los costos injustificados (fallas internas y externas) en cada proceso seleccionado para el monitoreo mensual del Hospital. Los datos simples de cada variable se registran en las casillas correspondientes al frente de cada variable en el estudio, en un formato estandarizado que sólo permite la digitación de la información en la casilla específica de fondo color amarillo, sin necesidad de puntos, comas o cualquier otro procesamiento. Se guarda y 
se entrega a la Dirección de calidad para su consolidación en la matriz de datos que va al sistema.

2. Una serie de matrices de procesamiento de la información registrada en cada matriz de datos, que automáticamente toma la información de la matriz de datos respectiva y calcula:

- Los costos directos de prevención en cada proceso.

- Los costos indirectos de prevención.

- Los costos directos de inspección.

- Los costos indirectos de inspección.

- Los costos directos de fallas internas en cada proceso.

- Los costos indirectos de las fallas internas.

- Los costos directos de fallas externas en cada proceso.

- Los costos indirectos de las fallas externas.

- Los costos de la hora y minuto del recurso humano interviniente en cada proceso, lo prorratea de acuerdo al número e ingresos de cada cargo y lo toma el aplicativo.

3. Las matrices de estándares en las cuales se define por la Gerencia y el directivo responsable de cada proceso, los recursos para el mantenimiento y mejoramiento de cada uno (inversión en prevención e inspección para la calidad) y el monto tolerable de costos de las fallas en cada proceso, lo cual se procesa y cuantifica automáticamente por el aplicativo, para generar en las matrices de resultado:

- Los costos estándares o aceptables para las actividades de prevención para la calidad (mantenimiento y mejoramiento de la calidad).

- Los costos estándares o aceptables para las actividades de inspección (control y auditoría), dirigidas ambas a entregar los resultados de cada proceso en las mejores condiciones y sin fallas.

Los costos estándares corresponden a aquellos recursos destinados por la empresa para la planeación, diseño, organización y mejoramiento con- tinuo de cada proceso, es decir, la inversión para que los resultados finales sean los adecuados y por ello se denominan "costos de calidad" (prevención e inspección).

- Igualmente, para las fallas internas y externas, se definen por la entidad unos costos aceptables o tolerables, los cuales para el proyecto se proponen inicialmente en el 10\% de la inversión en calidad. Lo ideal sería cero $(0 \%)$ porque no es razonable que se presenten fallas, pero también es cierto, que es imposible pensar en una organización perfecta que no presente fallas humanas y, por lo tanto, toda empresa acepta unos costos de no calidad tolerables, sobre los que se comparan los observados cada mes y que serían la meta a lograr con las estrategias de contención de costos.

4. Las matrices de resultado o tableros de costos de calidad y no calidad, en las cuales se entregan los resultados finales:

- Con el monto de la inversión en la prevención de cada proceso, es decir, el costo de los recursos están dirigidos al mantenimiento y mejoramiento del proceso con el fin de lograr resultados de excelente calidad sin errores.

- El monto de la inversión en inspección, es decir, el costo de los recursos invertidos en el control (auditoría) para que cada proceso termine satisfactoriamente como estaba programado y obtenga sus resultados sin fallas.

- El monto de los estándares o costo esperado por la empresa, de recursos destinados en cada proceso para las actividades de prevención e inspección, con el fin de tener un parámetro de comparación entre los establecido por la empresa y los costos reales observados en los recursos invertidos para la calidad del proceso.

- El monto de las fallas internas y externas observadas en cada proceso, es decir, el costo de la no calidad del pro- 
ceso, generado por las fallas (reintervenciones, reprocesos, correctivos. Investigaciones para resolver situaciones no deseadas, resolución de conflictos y fallas en la atención hospitalaria por ejemplo).

- El monto definido por la entidad como tolerable para dichos costos injustificados en cada proceso, tienen el fin de tener un parámetro de comparación de lo observado frente a lo tolerable por la empresa, y base para las metas de mejoramiento y contención de dichos costos.

5. Una matriz de recurso humano para el registro de cada uno de los cargos de planta y contrato por cada tipo de vinculación y categoría del cargo y asignación salarial u honorarios para el cálculo de los costos por hora y minuto de cada tipo de cargo. La información en esta matriz se diligencia una vez al inicio del sistema y sólo cuando exista una modificación en el número de cargos o el valor del ingreso.

6. El aplicativo toma cada número de cargos por categoría y asignación salarial u honorarios y prorratea los costos de acuerdo al ingreso de cada uno de los integrantes, para un costo del tiempo real en cada tipo de cargo.

Sin lugar a dudas, será un complemento fundamental del Sistema de gestión empresarial del Hospital. Le aporta el soporte del sistema con la cuantificación de dichos costos y evalúa mes a mes el impacto de las decisiones de control por proceso.

\section{Impacto}

Reconocido el monto de los costos invertidos en la calidad de los servicios por las empresas de salud y aquellos costos derivados de los problemas en los procesos claves de la empresa, se demostrará la importancia para la ges- tión empresarial, del conocimiento de esos costos injustificados, los cuales incurren mes a mes, permitirá que la medición sistemática le aporte a la gerencia de cada entidad los valores que se están destinando a garantizar la calidad, reduciendo los costos de no calidad en cada proceso con unos compromisos de gestión mensuales entre la gerencia y el jefe de cada repartición.

\section{Proyecciones del estudio}

1. El mercadeo del producto podrá hacerse a través de los mismos egresados de las especializaciones de Gerencia y de Auditoría en Salud, además de las direcciones locales y departamentales, directamente en las empresas de salud del país, al tener en cuenta que no existe un modelo acabado que realice la medición cuantitativa de los costos de calidad y de no calidad en las empresas del sector y la bibliografía existente. Adicional a los resultados de la presente investigación, se demuestra ampliamente que dichos costos son significativamente altos en la inmensa mayoría de las organizaciones, por lo que el conocimiento del monto mensual, será un factor de importancia para los compromisos de gestión en cuanto a la reducción de costos de operación de la empresa que cuente con este modelo desarrollado por la Universidad.

2. El estudio convierte la extensa bibliografía mundial sobre los costos de calidad y no calidad en una herramienta gerencial efectiva, confiable y de apoyo para la gestión empresarial.

3. La Universidad como formadora de gerentes y auditores para las empresas de salud en el Sistema General de Seguridad Social, les aporta a sus egresados y a las empresas del sector, un avance en los métodos de gestión empresarial y como producto generará ingresos propios para el desarrollo del área de investigación y del Centro de Consultoría. 


\section{Resultados de la investigación}

La primera medición realizada en un mes, seleccionado durante la ejecución del proyecto, demostró la validez de la hipótesis planteada, sobre la existencia de un monto significativo en dinero, considerado un gasto injustificado, debido a que corresponde a las acciones correctivas para solucionar los problemas en los procesos (costos de no calidad), como:

- Reprocesos.

- Repetición de atenciones.

- Repetición de facturas y cuentas.

- Resolución de quejas por problemas en el servicio a clientes externos.

- Fallas en los procesos contractuales.

- Costos por problemas de calidad en la atención hospitalaria de pacientes.

- Repetición y/o pérdida de exámenes de laboratorio clínico, no utilizados en el proceso asistencial.

- Repetición y/o pérdida de exámenes de radiología, no utilizados en el proceso asistencial.

- Reformulación y/o repetición de formulación de medicamentos.

- Costos financieros por mora en la recuperación de los dineros invertidos en la atención de los pacientes.

El proyecto logró el cumplimiento de los objetivos de la investigación y la entrega a los hospitales y a la Universidad de los productos programados:

1. La cuantificación de los montos invertidos en los procesos seleccionados, que sostienen el Sistema de calidad de la empresa, claves en el funcionamiento de la entidad.
2. La cuantificación de los costos generados por las fallas en los procesos claves seleccionados o costos de no calidad o injustificados en un mes de prueba.

3. El diseño de una metodología que logró crear un modelo de sistema para la medición sistemática de los costos de calidad y no calidad en la empresa mes a mes, como complemento trascendente del Sistema de Gestión Empresarial.

4. Ofrecer una propuesta concreta de proyección social mediante el desarrollo de una herramienta de mejoramiento de la gestión para las empresas del sector de la salud y la seguridad social en el país.

5. Favorecer el desarrollo del componente de investigación de los posgrados de Seguridad Social, con herramientas gerenciales de utilidad práctica para los egresados de Gerencia y Auditoría de Salud.

Como estaba programado, se realizó la medición de los costos de calidad y no calidad en un mes durante el desarrollo del proyecto en el Hospital Santa Clara de Bogotá D.C., empresa social del Estado, 3er nivel de complejidad, en la cual se encontró en septiembre de 2008, cifras significativas que justifican el monitoreo sistemático para la adopción de acciones de mejoramiento continuo, orientadas fundamentalmente a la contención de los costos injustificados en los procesos claves analizados.

La expresa decisión de la Gerencia de la Institución para el montaje del sistema de medición en el Hospital y la calidad profesional y humana observada en el equipo directivo de la entidad, fueron los principales factores de éxito para ello.

Los resultados del primer mes de cuantificación (septiembre de 2008) son presentados con las reservas en algunos procesos, para los cuales no fue posible obtener los datos en esta primera medición. Por algunas dificultades inter- 
nas del Hospital de Chapinero, el proyecto sufrió un retraso en su ejecución, pero los resultados preliminares demuestran similar importancia y fueron presentados en el mes de junio de 2009.

Una vez obtenida la información en las matrices de datos por procesos claves, seleccionados para la medición, se realizó automáticamente la cuantificación en las matrices de procesamiento diseñadas y construidas para el Sistema de medición de costos de calidad y no calidad durante el proyecto, luego de lo cual se produjeron las matrices de resultado que se presentan a continuación, con los costos de calidad en la parte superior de cada una, en la que se muestran los costos invertidos en la calidad de cada proceso en actividades de promoción y prevención para el mantenimiento y mejoramiento de cada proceso.

Comparativamente para los mismos procesos, en la parte inferior de la matriz aparecen los costos de no calidad observados, lo que posibilita la toma de acciones correctivas y/o de mejoramiento con cada responsable del proceso, convirtiéndose esto en los compromisos de gestión para la contención de los costos injustificados mes a mes, lo que debe producir como resultado, su reducción significativa y el mejoramiento de los resultados financieros del Hospital para su sostenibilidad:

Tabla 1. Matriz de resultados de costos de calidad y no calidad

Hospital Santa Clara ESE - Costos de calidad y no calidad

No. Septiembre de 2008

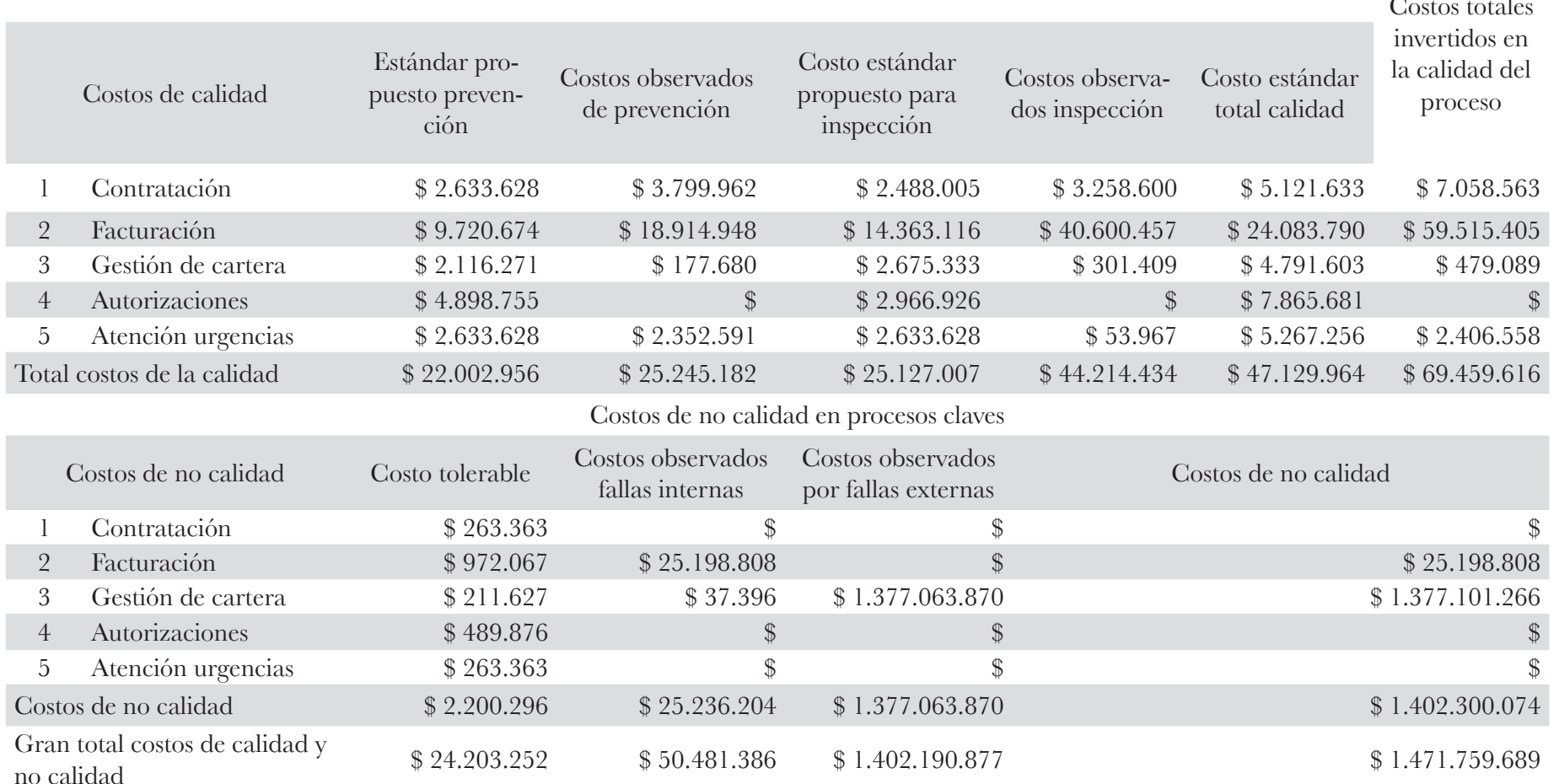

Costos de calidad en procesos claves 
La evaluación comparativa de la inversión en la calidad de cada proceso y los costos injustificados de cada mes, para cada uno de los mismos procesos, analizada mensualmente, permitirá, asimismo, evaluar el impacto de las decisiones tomadas en los meses anteriores.

Como puede observarse en la matriz de resultados de costos de calidad y no calidad en la tabla 1, la inversión de un poco más de 69 millones de pesos en el mantenimiento y mejoramiento de estos cinco procesos, con el fin de que todo salga perfectamente (costos de calidad), debería reflejarse mes a mes en la reducción significativa de los costos injustificados en dichos procesos. Lo observado demostró unos costos superiores a los 1.402 millones de pesos (costos de no calidad), fundamentalmente por los costos financieros (lucro cesante) de la cartera morosa del Hospital. Este es el reto para las directivas, que mes a mes, su reducción fue significativa. A su vez, estos resultados son también el aporte para la buena gestión, entregado por la Universidad al Hospital, como producto importante y trascendente de la investigación

En forma similar, se produce la Matriz de resultados para los otros cinco procesos claves seleccionados por la Gerencia del Hospital para la medición y la construcción del sistema:

Tabla 2. Matriz de resultados de costos de calidad y no calidad

\begin{tabular}{|c|c|c|c|c|c|c|c|}
\hline \multicolumn{8}{|c|}{ Hospital Santa Clara ESE - Costos de calidad y no calidad } \\
\hline No. & Septiembre de 2008 & \multicolumn{6}{|c|}{ Costos de calidad en procesos claves } \\
\hline & Costos de calidad & $\begin{array}{l}\text { Costo estándar } \\
\text { propuesto para } \\
\text { prevención }\end{array}$ & $\begin{array}{l}\text { Costos observados } \\
\text { de prevención }\end{array}$ & $\begin{array}{l}\text { Costo estándar } \\
\text { propuesto para } \\
\text { inspección }\end{array}$ & $\begin{array}{l}\text { Costos observa- } \\
\text { dos inspección }\end{array}$ & $\begin{array}{l}\text { Costo estándar } \\
\text { calidad }\end{array}$ & $\begin{array}{l}\text { Costos totales } \\
\text { invertidos en } \\
\text { la calidad del } \\
\text { proceso }\end{array}$ \\
\hline 6 & Laboratorio clínico & $\$ 1.274 .468$ & $\$ 21.548 .686$ & $\$ 789.356$ & $\$ 576.767$ & $\$ 2.063 .824$ & $\$ 22.125 .453$ \\
\hline 7 & Radiología & $\$ 867.614$ & $\$ 7.538 .651$ & $\$ 262.549$ & $\$ 476.282$ & $\$ 1.130 .163$ & $\$ 8.014 .933$ \\
\hline 8 & Hospitalización & $\$ 6.586 .996$ & $\$ 295.825$ & $\$ 3.270 .308$ & $\$ 8.953 .811$ & $\$ 9.857 .304$ & $\$ 9.249 .636$ \\
\hline 9 & Atención al usuario & $\$ 359.478$ & $\$$ & $\$ 292.963$ & $\$ 2.582 .787$ & $\$ 652.441$ & $\$ 2.582 .787$ \\
\hline 10 & Medicamentos & $\$ 4.674 .072$ & $\$ 5.285 .571$ & $\$ 797.900$ & $\$$ & $\$ 5.471 .973$ & $\$ 5.285 .571$ \\
\hline \multicolumn{2}{|c|}{ Total costos de la calidad } & $\$ 13.762 .629$ & $\$ 34.668 .733$ & $\$ 5.413 .076$ & $\$ 12.589 .647$ & $\$ 19.175 .705$ & $\$ 47.258 .380$ \\
\hline \multicolumn{8}{|c|}{ Costos de no calidad en procesos claves } \\
\hline & Costos de no calidad & Costo tolerable & $\begin{array}{l}\text { Costos observados } \\
\text { fallas internas }\end{array}$ & $\begin{array}{l}\text { Costos obser- } \\
\text { vados por fallas } \\
\text { externas }\end{array}$ & \multicolumn{3}{|c|}{ Costos de no calidad } \\
\hline 6 & Laboratorio clínico & $\$ 127.447$ & $\$ 127.941$ & $\$ 659.389$ & & & $\$ 787.330$ \\
\hline 7 & Radiología & $\$ 86.761$ & $\$ 41.308$ & $\$ 9.436 .626$ & & & $\$ 9.477 .935$ \\
\hline 8 & Hospitalización & $\$ 658.700$ & $\$ 274.727 .526$ & $\$$ & & & $\$ 274.727 .526$ \\
\hline 9 & Atención al usuario & $\$ 35.948$ & $\$ 4.025 .832$ & $\$ 507.978$ & & & $\$ 4.533 .810$ \\
\hline 10 & Medicamentos & $\$ 467.407$ & $\$ 1.362$ & $\$$ & & & $\$ 1.362$ \\
\hline \multicolumn{2}{|c|}{ Costos no calidad } & $\$ 1.376 .263$ & $\$ 278.923 .970$ & $\$ 10.603 .993$ & & & $\$ 289.527 .963$ \\
\hline \multicolumn{2}{|c|}{$\begin{array}{l}\text { Gran total costos de calidad y } \\
\text { no calidad }\end{array}$} & $\$ 15.138 .891$ & $\$ 313.592 .703$ & $\$ 16.017 .069$ & & & $\$ 336.786 .342$ \\
\hline
\end{tabular}


Cálculo de los costos de calidad y no calidad en empresas de salud y creación de un sistema de medición Luis Javier Arango C., Carlos Iván Rodríguez M., Rocío López C.

No obstante, la inversión superior a los 47 millones de pesos para el mantenimiento y mejoramiento de la calidad de los cinco procesos, se observaron costos injustificados (de no calidad) por más de 289 millones de pesos, los cuales de reducirse en su mayoría, darán un alivio financiero a la situación del Hospital y deberían mejorar la capacidad económica para el mejoramiento de la calidad en los servicios, como un valor agregado para el Hospital, la medición de los costos injustificados (de no calidad), observados al recibir y procesar las quejas de los clientes externos por los servicios recibidos, se midieron no sólo en forma general para el Hospital, sino que se analizaron por departamento, para facilitar la toma de decisiones específicas para un mayor impacto en el mejoramiento de cada área:

Tabla 3. Hospital Santa Clara - Costos de calidad y no calidad

\begin{tabular}{|c|c|c|c|c|c|}
\hline \multicolumn{6}{|c|}{ Hospital Santa Clara ESE - Costos de calidad y no calidad } \\
\hline No. & Septiembre de 2008 & \multicolumn{4}{|c|}{ Costos de calidad en procesos claves } \\
\hline Cost & os de calidad quejas cliente externo & Estándar & $\begin{array}{c}\text { Costos observados fallas } \\
\text { internas }\end{array}$ & $\begin{array}{l}\text { Costos observados por } \\
\text { fallas externas }\end{array}$ & $\begin{array}{l}\text { Costos de no calidad en } \\
\text { la atención al usuario }\end{array}$ \\
\hline 1 & Costo total quejas & $\$ 359.478$ & $\$ 4.025 .832$ & $\$ 503.643$ & $\$ 4.529 .475$ \\
\hline 2 & Costo quejas atención quirúrgica & $\$ 254.894$ & $\$ 0$ & $\$ 0$ & $\$ 0$ \\
\hline 3 & Costo quejas servicios clínicos & $\$ 509.787$ & $\$ 0$ & $\$ 0$ & $\$ 0$ \\
\hline 4 & Costo quejas UCI adultos & $\$ 127.447$ & $\$ 0$ & $\$ 0$ & $\$ 0$ \\
\hline 5 & Costo quejas UCI pediátrica & $\$ 127.447$ & $\$ 18.199$ & $\$ 0$ & $\$ 18.199$ \\
\hline 6 & Costo quejas cuidados intermedios & $\$ 254.894$ & $\$ 0$ & $\$ 0$ & $\$ 0$ \\
\hline \multicolumn{2}{|c|}{ Total costos de calidad } & & $\$ 4.044 .031$ & $\$ 503.643$ & $\$ 4.547 .674$ \\
\hline \multicolumn{6}{|c|}{ Costos de no calidad en procesos claves } \\
\hline & Costos de no calidad & Índice tolerable & $\begin{array}{l}\text { Costos observados fallas } \\
\text { internas }\end{array}$ & $\begin{array}{l}\text { Costos observados por } \\
\text { fallas externas }\end{array}$ & Costos de no calidad \\
\hline 1 & Costo total quejas & $\$ 1.376 .263$ & $\$ 4.025 .832$ & $\$ 503.643$ & $\$ 4.529 .475$ \\
\hline 2 & Costo quejas atención quirúrgica & $\$ 275.253$ & $\$ 0$ & $\$ 0$ & $\$ 0$ \\
\hline 3 & Costo quejas servicios clínicos & $\$ 550.505$ & $\$ 0$ & $\$ 0$ & $\$ 0$ \\
\hline 4 & Costo quejas UCI adultos & $\$ 137.626$ & $\$ 0$ & $\$ 0$ & $\$ 0$ \\
\hline 5 & Costo quejas UCI pediátrica & $\$ 137.626$ & $\$ 29.371$ & $\$ 0$ & $\$ 29.371$ \\
\hline 6 & Costo quejas cuidados intermedios & $\$ 275.253$ & $\$ 0$ & $\$ 0$ & $\$ 0$ \\
\hline 7 & Costo de las tutelas & $\$ 6.881 .314$ & & $\$ 0$ & $\$ 0$ \\
\hline \multicolumn{2}{|c|}{ Costos no calidad } & & $\$ 29.371$ & $\$ 503.643$ & $\$ 4.558 .846$ \\
\hline \multicolumn{2}{|c|}{ Gran total costos de calidad y no calidad } & & $\$ 4.073 .402$ & $\$ 1.007 .286$ & $\$ 9.106 .521$ \\
\hline
\end{tabular}


$\mathrm{Al}$ mirar la información, es evidente que no se tenían los datos específicos para la medición al momento de la cuantificación de los costos de calidad y no calidad, generados por la recepción y procesamiento de las quejas de los clientes externos por los servicios del Hospital.

Esta situación que se mejorará durante la implementación del sistema, una vez se logre la estandarización del manejo de la información y el registro real de los datos requeridos por el sistema.

Con la poca información obtenida se observó como la inversión superior a los 4,54 millones de pesos en prevención e inspección para la calidad del proceso, se evidenció que más de 4,55 millones como no justificados (de no calidad). La estandarización de la metodología logrará que la información futura sea más real.

\section{Conclusiones producto de la medición en el Hospital}

1. La medición realizada a los costos de calidad y no calidad, durante septiembre de 2008, demostró que las fallas en los procesos generan unos costos injustificados (de no calidad), significativamente altos a pesar de tratarse del primer mes de medición, en el que no pudieron obtenerse todos los datos requeridos.

2. El conocimiento sobre los costos de inversión en calidad para lograr la óptima calidad de los procesos y aquellos generados por las fallas o costos de no calidad, mes a mes, se convierte en un importante apoyo para la toma de decisiones de mejoramiento y control, dado que presenta en forma cuantitativa el monto de la inversión en calidad y costos injustificados de no calidad.

3. El sistema de costos de calidady no calidad será un trascendente complemento al Sistema de gestión empresarial para el Hospital y luego de su implementación, se convertirá en una herramienta de gestión de muy fácil manejo por el mínimo procesamiento que requiere del personal del Hospital. El sistema se deja instalado e implementado para los diez procesos claves seleccionados para el desarrollo del estudio. Una vez implementado, la entidad podrá adicionar procesos para el monitoreo si lo considera pertinente.

\section{Recomendaciones}

Con el fin de que el Sistema de costos de calidad y no calidad mantenga la información confiable y oportuna sobre las variables en estudio, es importante tener en cuenta las siguientes recomendaciones para la entidad que implemente el sistema:

1. Realizar la difusión en el personal directivo y la suficiente motivación al responsable de cada proceso, con el fin de que participe activamente en el registro de los datos indispensables para la medición de los costos de calidad y no calidad, en el análisis de los resultados y en la toma de decisiones para la reducción de los costos injustificados de los procesos.

2. Estandarizar el uso de la matriz de datos, de manera que en los primeros cinco días de cada mes se entregue la información de cada área responsable del proceso, ubicada en el escritorio de cada uno, con el fin de que sólo tengan que abrirlo seleccionando el ícono del sistema. Un auxiliar de confianza puede diligenciarla y entregar el archivo en la dirección de calidad del Hospital, para su inclusión en el sistema.

Luego de los primeros meses de operación del sistema, las matrices de datos se pueden integrar al Sistema de información del Hospital, con el fin de que los datos se tomen automáticamente de los informes regulares que realiza el área, con lo cual se minimiza el tiempo requerido de trabajo del personal del Hospital en el Sistema de costos de calidad y no calidad. 
3. Redefinir los estándares de dos a tres meses luego de la implementación del sistema, con el fin de ajustarlos a las reales condiciones de los procesos en la entidad y convertirlos en metas para cada período.

4. Los costos en el primer mes de medición no corresponden a la realidad muy seguramente, debido a que muchos de los datos son imposible de obtener con la precisión necesaria, situación que era predecible por tratarse de un sistema nuevo de medición. Una vez se estandaricen los datos necesarios para el monitoreo de cada proceso, la información será más completa y confiable.

5. Como estrategia trascendente para lograr la sostenibilidad financiera del Hospital, la realización de un plan para la contención de los costos de operación, tendrá en el sistema de costos de calidad y no calidad, un soporte importante para la reducción de los costos injustificados en los procesos claves de la entidad.

6. Una vez implementado el sistema y estandarizado el registro y procesamiento de la información sobre los procesos seleccionados para la primera fase de medición de los costos de calidad y no calidad, se podrá adicionar el seguimiento de otros procesos de la entidad, con una metodología similar, con lo cual se obtendrá una medición más integral de los costos del Hospital en calidad y no calidad.

7. El programa de implementación del sistema preparado por el equipo de investigación de la Universidad, como un soporte para la entidad, facilitará la estandarización de la metodología y capacitación del personal. Durante los tres primeros meses luego de la finalización del estudio, se apoyará directamente por el propio grupo de investigación como estaba programado desde el inicio del proyecto.

8. Con el proyecto, la Universidad cuenta con un producto para el apoyo a la gestión de los egresados de las especializaciones de Gerencia de Instituciones de Se- guridad Social en Salud y de Auditoría en Salud, que servirá para la formación de los estudiantes y será un proyecto para ser desarrollado en otras empresas de salud y seguridad social del país, a través del Centro de Consultoría de la Dirección de Seguridad Social.

Finalmente, el Sistema de costos de calidad y no calidad, creado con el proyecto, es una metodología de costos por proceso, que permite la inclusión de procesos para el monitoreo, lo cual es un avance importante en la gestión empresarial, dado que en el país en general, no se ha desarrollado un sistema de costos por proceso para empresas de salud.

Las matrices de procesamiento y producción de resultados del sistema de costos de calidad y no calidad, han estandarizado el procedimiento de cuantificación de dichos costos, por lo que simplemente, cada proceso adicional sólo requiere que se le puedan identificar las variables para la medición de la inversión en calidad (prevención e inspección) y aquellas que contengan información sobre los recursos invertidos en la corrección de las fallas en los procesos internos y externos

\section{Referencias}

Alexander, A. (1994). La mala calidady su costo. Buenos Aires: Editorial Addison Wesley Iberoamericana.

Alexander, A. (1994). La mala calidad y su costo. Wilmington, Delaware, U.S.A.: Addison-Wesley Iberoamericana.

Barrie, D. (1993). Los costos de la calidad (1 $1^{\text {a }}$ ed.). México: Editorial Grupo Editorial Iberoamérica de México.

Blanco, L. (1999). Productividad. Factor estratégico de competitividad a nivel global. Bogotá: Escuela Colombiana de Ingeniería. 
Besterfield, D. (1995). Control de calidad. México: Editoral Prentice Hall Hispanoamericana.

Campanenella, J. (1992). Principios de los costos de la calidad. Madrid: Ediciones Díaz de Santos.

Castañeda, G. (1999). Manual de calidad para la pequeña y mediana empresa. México: Universidad Iberoamericana. Dirección de Difusión Universitaria.

Del Río, C. (2000). Costo Integral Conjunto: el método más actualizado, revolucionario y práctico (2a ed.). México: Editorial Ecafsa.

Deming, E. (1989). Calidad, productividad y competitividad. La salida de la crisis. Madrid: Ediciones Díaz de Santos.

García, M. (1994). Cultura de la calidad (1ª ed.). Lima: Editorial UNMSM.

Grima, P. \& Tort-Martorell, J. (1995). Técnicas para la gestión de la calidad Madrid Ediciones: Díaz de Santos.

González, J. \& Izar, J. (2004). Las 7 herramientas básicas de la calidad: descripción de las 7 herramientas México: Universidad Autónoma de San Luis de Potosí. Unidad Académica

Harrington, J. (1987). El costo de la mala calidad (1 ${ }^{\mathrm{a}}$ ed.). España: Ediciones Díaz de los Santos.
Heizer, J. \& Render, B. (1997). Dirección de la producción. Decisiones estratégicas. Madrid: Editoral Prentice Hall Hispanoamericana.

Horngren, C., Foster, G. \& Datar, S. (1996). Contabilidad de costos. Un enfoque de gerencia. México: Editorial Prentice Hall Hispanoamericana.

Imai, M. (1998). Cómo implementar el kaizen en el sitio de trabajo (Gemba). Bogotá: Editorial McGraw-Hill Interamericana.

Juran, J. (1990). Furan y la planificación para la calidad. Madrid: Ediciones Díaz de Santos.

Multidisciplinaria Zona Media. San Luis Potosí. S.L.P.

Naveh, E. \& Halevy, A. (1996). El costo de la no calidad, 20 (03).

Rincón, H. (2000). Cruzando fronteras: tendencias de contabilidad directiva para el siglo XXI. En Sistemas de costos. Convencional y actual. Mérida: Publicación de la Facultad de Ciencias Económicas y Sociales. Universidad de los Andes.

Ruelas, E. (1993). Calidad, productividad y costos. En $R e^{-}$ vista de Salud Pública, Vol. 35, No. 3, México.

Terrazas, M. (1998). Diseño de un sistema de costos de calidad. Editorial San Pedro Garza García.

Toro, F. (1990). Costos y presupuestos con base en tareas. Madrid. 